\title{
'Enseñar a ser médicos': un análisis de opinión de los médicos implicados en la docencia de la clínica práctica (I). Conclusiones del análisis cualitativo y metodología para un estudio cuantitativo
}

\author{
Jesús Millán Núñez-Cortés, José Antonio Gutiérrez-Fuentes
}

Resumen. La enseñanza de la medicina en el momento actual debe centrarse de forma primordial en las competencias
clínicas propias del médico, que le permitan un ejercicio profesional adecuado. En este sentido, el entorno clínico es el lu-
gar idóneo para la enseñanza-aprendizaje de tales competencias. Sin embargo, la enseñanza de la medicina clínica a tra-
vés de la enseñanza de clínica práctica tiene, a día de hoy, limitaciones que deben identificarse y que coexisten con forta-
lezas, las cuales deben fomentarse. En este trabajo se presentan los primeros datos de 'Enseñar a ser médicos', un estudio
trasversal realizado en el ámbito hospitalario de los centros asociados a la Universidad Complutense de Madrid. Se pre-
senta el análisis cualitativo de la opinión de los profesionales implicados en la enseñanza de la práctica clínica, mediante
una encuesta estructurada y precodificada, con preguntas abiertas y semiabiertas. Entre las debilidades detectadas cabe
citar: el excesivo componente teórico de la enseñanza, algunas carencias formativas específicas (relaciones interprofesio-
nales y comunicación), el escaso fomento de la investigación, el mantenimiento del examen de médico interno residente
(MIR) como referente y la escisión entre las funciones docentes y asistenciales en el entorno hospitalario. Entre las fortale-
zas de la enseñanza actual: la elevada preparación del alumno, su elevado rendimiento académico y, de forma significati-
va, el inicio de cambios innovadores en el enseñanza de la medicina.

Palabras clave. Debilidades. Enseñanza práctica. Fortalezas. Práctica clínica.

'Teaching how to be a doctor': an analysis of the opinions of doctors involved in the teaching of clinical practice (I). Conclusions from the qualitative analysis and methodology for a quantitative study

Summary. Today, the teaching of medicine must focus primarily on the clinical competencies expected of a physician, which enable them to practise their career in an adequate manner. In this regard, the clinical setting is the ideal place to teach-learn such competencies. Yet, the teaching of clinical medicine through the teaching of clinical practice has, as we stand today, its drawbacks, which must be identified, and also its strengths, which need to be fostered. In this work we present the first data from 'Teaching how to be a doctor', a cross-sectional study conducted in the hospital setting in centres associated with the Universidad Complutense in Madrid. We report the qualitative analysis of the opinions of professionals involved in the teaching of clinical practice, which was carried out by means of a structured and pre-coded survey, with both open-ended and semi-open-ended questions. The weaknesses that were detected include: the excessive theoretical component of the teaching, some specific shortcomings in training (interprofessional relationships and communication), scarce promotion of research, the continued presence of the medical resident exam (MIR) as a referent and the rift between teaching and healthcare duties in hospitals. Some of the strengths of the current teaching practice include: students are very well prepared on finishing their training, they have a high level of academic achievement and, significantly, we are beginning to see some innovative changes in the way medicine is taught.

Key words. Clinical practice. Practical teaching. Strengths. Weaknesses.
Departamento de Medicina. Universidad Complutense. Cátedra de Educación Médica Fundación Lilly-Universidad Complutense. Madrid, España.

Correspondencia: Prof. Jesús Millán Núñez-Cortés. Departamento de Medicina. Universidad Complutense. Facultad de Medicina. Ciudad Universitaria. E-28040 Madrid.

E-mail:

jesus.millan@madrid.org

Conflicto de intereses: No declarado.

Conflict of interests: None declared.

(c) 2012 Educación Médica 


\section{Introducción}

Para cumplir con el ejercicio profesional de la medicina, el médico debe ser consciente de que adquiere una triple responsabilidad: frente a sí mismo, frente a los enfermos y frente a la sociedad. Desde este punto de vista, sólo la adquisición de todo un conjunto de conocimientos (saberes) y valores puede garantizar un adecuado ejercicio de la profesión médica, que se debe constituir como un elemento fundamental del sistema sanitario.

El médico debe tener y mantener, en todo momento, un nivel actualizado de conocimientos, unas habilidades clínicas y una conducta acordes con esas responsabilidades. Adicionalmente se espera del médico que sea responsable y buen gestor de los medios que se le brindan en el desarrollo de su actividad al servicio del paciente, incluso en situaciones en las que su función y desempeño profesional pueden no ser comprendidas ni apreciadas en su justa dimensión.

Por todo ello es una responsabilidad del personal implicado en la enseñanza de la medicina, y consustancial con esta enseñanza, reflexionar cómo y de qué manera se debe definir el escenario donde tiene lugar dicha enseñanza y cuáles han de ser los elementos que definan el papel del médico con responsabilidad para formar a otros médicos; esto es, analizar el entorno y los actores que participan en la enseñanza de la medicina.

En el momento actual, de profundo cambio, cabe preguntarse cómo podemos llegar a mejorar la enseñanza de la medicina de acuerdo con los nuevos principios más básicos, cómo ha cambiado la forma de transmitir el conocimiento médico y el escenario en el que debe practicarse, cuáles son los valores y los compromisos del estudiante y del profesor, cuáles son las necesidades de ambos, o si estamos -o no- interesados por la innovación en materia educativa e incorporamos estrategias acorde con ello.

El presente estudio se diseñó asumiendo que la enseñanza de la medicina es propia del quehacer diario de los médicos en el entorno clínico, y que los médicos empiezan a serlo -en una gran parteuna vez que comienza su formación clínica de la mano de otros médicos.

En consecuencia, el estudio 'Enseñar a ser médicos' tiene como objetivo primario explorar la opinión de los médicos implicados en la docencia de la medicina, específicamente en el entorno de la práctica clínica, a fin de conocer la valoración de ésta, la actitud frente a situaciones reales actuales, las prioridades y las posibles mejores prácticas.
En este artículo (parte I) se presentan los resultados de la valoración cualitativa de un grupo de médicos implicados en la enseñanza de la medicina clínica, y derivado de dichos resultados, el diseño de la muestra y del instrumento (encuesta) para valorar de forma cuantitativa la enseñanza de la medicina práctica en su visión actual (parte II) y en las perspectivas futuras (parte III).

\section{Metodología}

Se trata de un estudio transversal, utilizando información a través de un instrumento tipo encuesta, realizado por la Cátedra de Educación Médica Fundación Lilly-Universidad Complutense, con la ayuda de una empresa experta en estudios sociológicos (Metroscopia).

Para el análisis cualitativo, se elaboró en primer lugar un cuestionario para obtener información relevante procedente de responsables de la enseñanza clínica de la medicina en hospitales universitarios. En dicho cuestionario se incluían aspectos sobre el grado de satisfacción con el sistema actual de formación de médicos y las supuestas carencias, deficiencias o disfunciones que perciben en él, lo que podría permitir detectar las fortalezas y las debilidades del sistema.

Los resultados que se muestran en el presente trabajo son los extraídos de un análisis cualitativo mediante entrevistas en profundidad, estructuradas con el cuestionario prediseñado. Dichas entrevistas se produjeron a través de un diálogo abierto con un grupo de médicos con clínicos con responsabilidad docente. Dicho grupo estaba compuesto por tres catedráticos, tres profesores titulares y tres profesores asociados o colaboradores, que estaban muy directamente involucrados en la enseñanza práctica sanitaria en tres hospitales universitarios vinculados a la Universidad Complutense de Madrid durante el curso 2008-2009.

La entrevista fue llevada a cabo por un sociólogo experimentado, obteniendo información cualitativa detallada al permitir a la persona entrevistada manifestarse sobre los temas en cuestión, pero sin las rigideces de un cuestionario cerrado. La selección del grupo no pretendía la estricta representatividad estadística, sino más bien obtener, de un número limitado de profesionales, un repertorio argumental sobre los ítems valorados que fuera lo más amplio y matizado posible. Con ello se pretendía posteriormente diseñar una encuesta amplia sobre los distintos aspectos, y que se pudiera aplicar a una población más extensa de personas de distintos estamen- 
Tabla I. Debilidades y fortalezas de la enseñanza médica actual.

Debilidades
Enfoque teórico excesivo
Necesidad de adaptar programas teóricos
Algunas carencias formativas específicas: relaciones y comunicación
Escaso fomento de la investigación
Examen MIR como referente
Escisión docencia/asistencia
Resistencia al cambio

\section{Fortalezas}

Alumno con elevada preparación

Alumnos con alto rendimiento académico

Programas teóricos completos

Inicio de cambios e innovación educativa

tos implicados en la docencia práctica en los hospitales universitarios (partes II y III del estudio).

\section{Resultados}

En este primer trabajo se presentan los resultados del análisis cualitativo obtenido en la primera parte del estudio.

Del análisis de las opiniones del grupo evaluado se desprende que la enseñanza actual de la medicina tiene una serie de fortalezas y debilidades, de las cuales las fundamentales se reflejan en la tabla I.

En la tabla II se recogen las distintas categorías sobre las que se estructuró la encuesta posterior para el estudio cuantitativo, y que incluye los aspectos clave derivados del análisis cualitativo, en un intento de extraer los elementos esenciales reflejados por el análisis previo y que pueden definir las características de la enseñanza de la clínica en la actualidad.

Posteriormente se obtuvo una muestra estadísticamente significativa de aquellos médicos que asumen o tienen asignada algún tipo de docencia de práctica clínica a estudiantes de medicina en los tres hospitales vinculados a la Universidad Complutense de Madrid. En esta muestra se incluyeron no sólo docentes-funcionarios (catedráticos y titulares), sino también profesores asociados y colaboradores, y otro personal médico sin ninguna obligación formal
Tabla II. Cuestionario estructurado para la encuesta del estudio cuantitativo.

\begin{tabular}{l}
\hline Datos demográficos \\
\hline Preguntas sobre la enseñanza actual \\
Valoración de la enseñanza actual de la medicina en su conjunto \\
Valoración de la enseñanza práctica \\
Factores positivos y negativos en la enseñanza \\
Enseñanza de distintas áreas competenciales \\
Administración de recursos y medios para la docencia
\end{tabular}

Preguntas sobre el profesorado

Satisfacción de la labor docente

Reconocimiento de la labor docente

Motivación como docente

Implicación del personal docente con o sin reconocimiento formal en la enseñanza de la medicina

Nivel de implicación en el proceso educativo

Tiempo de dedicación a la actividad docente

Valoración de la docencia y la investigación en la carrera profesional Interés por la participación en estrategias innovadoras

Preguntas sobre el alumnado

Nivel de conocimiento y preparación alcanzados por los alumnos antes de ingresar en la universidad

Formación obtenida al finalizar la carrera

Enseñanza práctica de los alumnos

Importancia de las actividades prácticas

Duración de las actividades prácticas

Factor clave para la integración del alumno en los servicios clínicos

docente, pero que en la práctica la asumen (tanto médicos de plantilla como médicos residentes).

Sobre la base del año 2009, los tres hospitales universitarios considerados tenían en total una plantilla aproximada de 4.950 personas, de las cuales 44 eran catedráticos de universidad, 68 eran profesores titulares de universidad y unos 595 eran profesores asociados de Ciencias de la Salud. Los médicos internos residentes sumaban en total 1.480 y el resto de médicos sin responsabilidades docentes reconocidas representaban una cifra que cabía estimar en 2.763 personas. La composición total de 
Tabla III. Distribución de la muestra para el estudio cuantitativo sobre la enseñanza de la medicina práctica en hospitales.

\begin{tabular}{lcccc}
\hline & $\begin{array}{c}\text { Hospital A } \\
(n=126)\end{array}$ & $\begin{array}{c}\text { Hospital B } \\
(n=161)\end{array}$ & $\begin{array}{c}\text { Hospital C } \\
(n=148)\end{array}$ & $\begin{array}{c}\text { Total } \\
(n=435)\end{array}$ \\
\hline Catedráticos & 3 & 24 & 10 & $37(8,5 \%)$ \\
\hline Profesores titulares & 3 & 29 & 19 & $51(11,6 \%)$ \\
\hline Profesores asociados & 65 & 65 & 66 & $196(45 \%)$ \\
\hline Médicos internos residentes & 33 & 19 & 25 & $77(17,7 \%)$ \\
\hline Personal no docente & 22 & 24 & 28 & $74(17 \%)$ \\
\hline
\end{tabular}

la muestra sobre la que finalmente se llevaría a cabo la entrevista estructurada fue de 435 médicos (126 médicos ejercían su labor docente en el hospital A, 161 en el hospital B y 148 en el hospital C). Para el análisis global y de conjunto de los resultados, los datos fueron objeto de ponderación para restituir el peso proporcional real, en función de la distribución de médicos docentes de práctica clínica, en los tres hospitales vinculados a la universidad. Los porcentajes de personas encuestadas sobre el total de pertenecientes a su colectivo fueron: $84 \%$ de catedráticos, $75 \%$ de profesores titulares, $33 \%$ de profesores asociados, $3 \%$ de médicos de plantilla sin vínculo docente y $5 \%$ de médicos internos residentes. Para un intervalo de confianza del 95,5\%, y asumiendo los principios del muestreo aleatorio simple, en la hipótesis más desfavorable de máxima indeterminación ( $p=q=50 \%$ ), el error de muestreo que corresponde a los datos referidos al total de la muestra es de $\pm 4,6 \%$.

En la tabla III se exhiben los resultados finales de la muestra seleccionada para el análisis cuantitativo posterior con la aplicación de la encuesta diseñada (estructurada y precodificada, con preguntas abiertas y semiabiertas), permitiendo así analizar por separado las respuestas de los cinco grupos de médicos con docencia clínica: catedráticos, profesores titulares, profesores asociados, personal no docente y médicos internos residentes.

\section{Discusión}

En el presente estudio, entre las fortalezas que se identifican en la enseñanza actual de la medicina hay que subrayar la presencia de alumnos en el grado con una elevada preparación y un alto rendi- miento académico. Y eso va unido a la existencia de unos programas teóricos completos. Del mismo modo se destaca el inicio de la innovación educativa y la orientación a la adquisición de competencias clínicas [1]; su definición, el entrenamiento para su adquisición y las evaluaciones de las competencias [2] son puntos fuertes que deben tenerse en consideración en el momento actual.

Sin embargo, el estudio destaca como una de las debilidades de la enseñanza médica más importante su enfoque teórico excesivo. Se siguen impartiendo excesivos contenidos teóricos y en muchos casos irrelevantes, de escasa utilidad para nuestros estudiantes en el momento de iniciar su práctica profesional, sobre todo si tenemos en cuenta la velocidad con la que se generan nuevos conocimientos científicos. La enseñanza impartida sigue estando orientada sobre todo a dar información. Es una enseñanza todavía centrada en el profesor, en vez de centrarse en el aprendizaje del estudiante, y no se dirige a que éste sea capaz de adquirir el hábito del aprendizaje autónomo. Se sigue empleando una metodología poco activa y basada en la lección magistral. Las metodologías de evaluación que se utilizan se centran en la evaluación de los conocimientos teóricos. Sigue persistiendo una frontera infranqueable entre el período preclínico y clínico que impide una integración real básica-clínica.

La mejora de la enseñanza conlleva unos cambios necesarios en la actividad docente actual que se pueden ver obstaculizados por unas barreras para la implantación de nuevos modelos, como son las relacionadas con la implicación del profesorado: la falta de reconocimiento de su labor docente por parte de las instituciones, la necesidad de estímulo -no sólo económico- y la resistencia al cambio.

Hasta ahora, la función docente se considera, en general, como poco valorada. Existe una clara falta de motivación de una gran parte del profesorado debido a la ausencia de su reconocimiento tanto en el ámbito sanitario donde ejercen su labor como en la universidad. Esta falta de reconocimiento se detecta en el ámbito académico y en el nivel económico. En consecuencia, la enseñanza puede quedar dañada por falta de motivación, de forma que las personas afectadas no se impliquen más y mejor en la mejora del proceso docente.

Por último, de acuerdo con el análisis cualitativo del grupo encuestado, los actuales planes de estudio no tienen en cuenta los valores del profesionalismo [3]. A este respecto, los encuestados han destacado una falta evidente de recursos humanos cualificados, así como de recursos materiales para introducir cambios reales en esta dirección. 
Además, la opinión de los profesores encuestados coincide en que la enseñanza actual se encuentra excesivamente orientada a la consecución de resultados óptimos en el examen de médico interno residente, y por ello algunas áreas competenciales (p. ej., relaciones interprofesionales y comunicación) suelen ser deficitarias, de tal manera que el fomento y la formación en investigación resultan escasos.

Un aspecto particularmente importante es que, en el ambiente formativo de la medicina clínica (en el ámbito del hospital universitario), se detecta una escisión entre la docencia y la asistencia que repercute negativamente sobre la formación del futuro médico. Paralelamente, la docencia práctica, si bien se ha incrementado en cantidad, no ha mejorado excesivamente en calidad, y las actividades prácticas tanto básicas como clínicas adolecen en ocasiones de falta de pertinencia o de exceso de pasividad por parte del estudiante. Por tanto, el clima educativo en el entorno clínico tiene un gran margen de mejora.

A nuestro juicio, se hace necesaria la optimización de nuevas estrategias orientadas a la adquisición de competencias clínicas con participación de todos los involucrados en el proceso educativo [4-6]. Esto obliga a predefinirlas, es decir, conceptualizar bien aquello que entendemos que todos los alumnos deben conocer y saber hacer a su paso por las facultades de medicina, desarrollar estrategias para que el alumno se entrene en ellas e implantar modelos de evaluación objetiva -evaluación clínica objetiva y estructurada (ECOE)- [7].

Por ello, el presente estudio parece destacar algunos elementos clave para llevar a cabo una enseñanza práctica de calidad, como son la existencia de un buen docente [8], un plan de asistencia regular y suficiente (en términos de temporalidad), la actitud e interés del alumno, los conocimientos previos, la participación del alumno en las actividades cotidianas de la práctica clínica y, lógicamente, la posibilidad de realizar actividades ante el paciente (en la zona de hospitalización, consultas, bloque quirúrgico, urgencias, consultas de atención primaria, etc.) [9].

En consecuencia, la amplia y profunda dedicación del alumno a la práctica clínica permitiría establecer modelos de integración del alumno en los servicios clínicos, requiriendo para ello la motivación del estudiante y del profesor, la colaboración de todo el servicio clínico, la incorporación real del alumno a las actividades con asignación de tareas con responsabilidad predefinidas, la tutoría y supervisión de las actividades, así como una correcta proporción entre el número de alumnos y los recursos humanos (profesorado) y materiales. El papel del alumno adquiriría una nueva dimensión como protagonista de su autoaprendizaje [10]: el alumno se sometería a un proceso con el objeto de aprender tanto a nivel teórico como práctico [11], con la conciencia de que debe poner para ello el máximo empeño volitivo y toda la capacidad perceptiva, racional y relacional que posea con el fin de aprovechar al máximo los recursos puestos a su disposición.

\section{Bibliografía}

1. Millán Nuñez-Cortés J. Las competencias clínicas. Educ Med 2005; 8: S13-14.

2. Malone K, Supri S. A critical time for medical education: the perils of competence-based reform of the curriculum. Adv Health Sci Educ Theory Pract 2012; 17: 241-6.

3. Clauser BE, Margolis MJ, Holtman MC, Katsufrakis PJ, Hawkins RE. Validity considerations in the assessment of professionalism. Adv Health Sci Educ Theory Pract 2012; 17: 165-81.

4. Dressler DD, Pistoria MJ, Budnitz TL, McKean SC, Amin AN. Core competencies in hospital medicine: development and methodology. J Hosp Med 2006; 1: 48-56.

5. McKean SC, Budnitz TL, Dressler DD, Amin AN, Pistoria MJ. How to use the core competencies in hospital medicine: a framework for curriculum development. J Hosp Med 2006; 1 (Suppl 1): 57-67.

6. Albanese MA, Mejicano G, Anderson WM, Gruppen L. Building a competency-based curriculum: the agony and the ecstasy. Adv Health Sci Educ Theory Pract 2010; 15: 439-54.

7. Harden R, Glesson F. Assessment of clinical competence using an objective structured clinical examination (OSCE). Med Educ 1979; 13: 41-54.

8. Higgs J, McAllister L. Being a clinical educator. Adv Health Sci Educ Theory Pract 2007; 12: 187-200.

9. Millán Núñez-Cortés J, García-Seoane J, Calvo-Manuel E, Díez-Lobato R, Calvo-Manuel F, Nogales-Espert A. Relaciones de la facultad de medicina con el hospital universitario. La enseñanza de la medicina clínica. Educ Med 2008; 11: 3-6.

10. Alaminos M, Campos-Sánchez A, Caracuel MD, RodríguezMorata A. Modelos didácticos para el autoaprendizaje. Actual Med 2009; 94: 49-53.

11. Van de Wiel MW, Van den Bossche P, Janssen S, Jossberger H. Exploring deliberate practice in medicine: how do physicians learn in the workplace? Adv Health Sci Educ Theory Pract 2011; 16: 81-95. 